\title{
Molecular characterization of a thaumatin-like protein PR-NP24 in tomato fruits
}

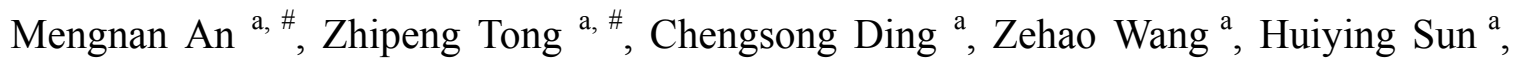
Zihao Xia ${ }^{\mathrm{a}}$, Mingfang $\mathrm{Qi}^{\mathrm{b}}$, Yuanhua $\mathrm{Wu}^{\mathrm{a}}$, Yue Liang ${ }^{\mathrm{a} *}$

${ }^{a}$ College of Plant Protection, Shenyang Agricultural University, Shenyang 110866, China

${ }^{\mathrm{b}}$ College of Horticulture, Shenyang Agricultural University, Shenyang 110866, China

Correspondence

E-mail: yliang@syau.edu.cn

\# Equal contribution 


\section{Supporting information}

Table S1 Primers used for constructs generation and RT-qPCR analysis.

\begin{tabular}{ll}
\hline Primer & Sequences $\left(5^{\prime}-3^{\prime}\right)$ \\
\hline TRVNP24+ & CCTCCATGGGGATCCTTTCAACAAACATGGGCTAC \\
TRVNP24- & CGAGACGCGTGAGCTCCCTGCAGCATTAAAGTTGC \\
qNP24+ & CCTTGGACCAGTTTAGCAAC \\
qNP24- & ACATGGACCTTGGGTGCAAC \\
qActin+ & CGGTGACCACTTTCCGATCT \\
qActin- & TCCTCACCGTCAGCCATTTT \\
\hline
\end{tabular}


Table S2 List of proteins identified from the different fruits.

\begin{tabular}{|c|c|c|c|c|c|}
\hline Plants & Tissue & Accession & Score & $\operatorname{Cov} \%$ & Peptides \\
\hline \multirow[t]{17}{*}{ Micro-Tom } & exocarp & P12670 & 327 & 37.7 & GQTWVINAPR \\
\hline & & & & & TGCNFNAAGR \\
\hline & & & & & CHAIHCTANINGECPR \\
\hline & & & & & CPDAYSYPQDDPTSTFTCPGGSTNYR \\
\hline & & & & & VPGGCNNPCTTFGGQQYCCTQGPCGPTELSK \\
\hline & flesh & P12670 & 302 & 31.7 & GQTWVINAPR \\
\hline & & & & & TGCNFNAAGR \\
\hline & & & & & CHAIHCTANINGECPR \\
\hline & & & & & NNCPYTVWAASTPIGGGR \\
\hline & fruit & P12670 & 1040 & 46.6 & GQTWVINAPR \\
\hline & & & & & TGCNFNAAGR \\
\hline & & & & & NNCPYTVWAASTPIGGGR \\
\hline & & & & & CHAIHCTANINGECPR \\
\hline & & & & & CPDAYSYPQDDPTSTFTCPGGSTNYR \\
\hline & & & & & RCPDAYSYPQDDPTSTFTCPGGSTNYR \\
\hline & & & & & VPGGCNNPCTTFGGQQYCCTQGPCGPTELSK \\
\hline & & & & & ALKVPGGCNNPCTTFGGQQYCCTQGPCGPTELSK \\
\hline \multirow[t]{8}{*}{ Fentailang } & exocarp & P12670 & 1335 & 21.9 & GQTWVINAPR \\
\hline & & & & & TGCNFNAAGR \\
\hline & & & & & NNCPYTVWAASTPIGGGR \\
\hline & & & & & CHAIHCTANINGECPR \\
\hline & flesh & P12670 & 184 & 8.1 & GQTWVINAPR \\
\hline & & & & & TGCNFNAAGR \\
\hline & fruit & P12670 & 37 & 8.1 & GQTWVINAPR \\
\hline & & & & & TGCNFNAAGR \\
\hline tamarillo & exocarp & P07052 & 49 & 4.4 & TNCNFDGSGR \\
\hline potato & exocarp & P07052 & 65 & 4.4 & TNCNFDGSGR \\
\hline black nightshade & exocarp & P25871 & 36 & 4.0 & TNCNFDGAGR \\
\hline
\end{tabular}




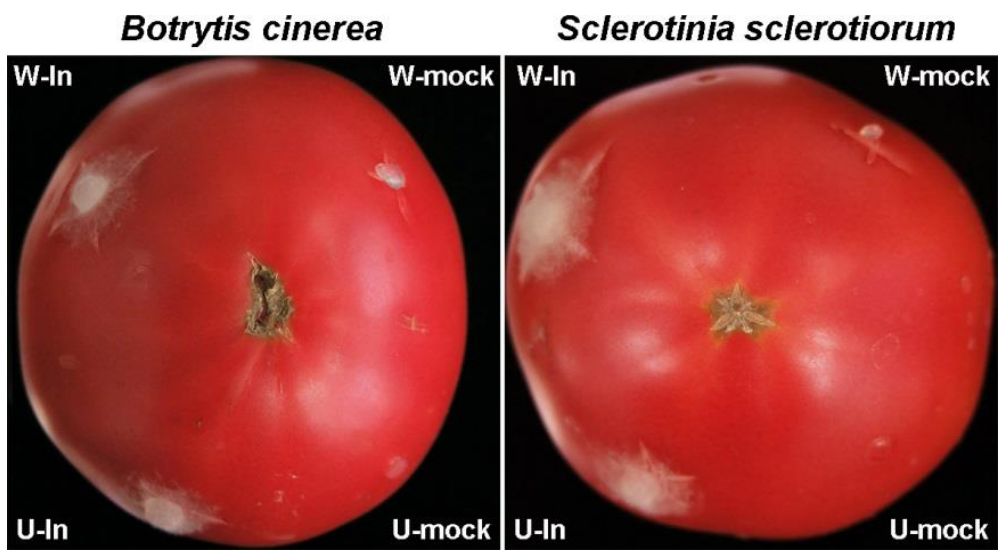

Figure S1 Symptoms of wounded (W-In) and unwounded (U-In) tomato inoculated by Sclerotinia sclerotiorum at 24 hours after inoculation (hai) and Botrytis cincerea at 48 hai while the PDA plugs served as the corresponding mocks (W-mock and U-mock). 